\title{
Emergency department treatment plans
}

\section{Christine East}

Background: A small population of "frequent visitors" make up a disproportionate number of ED visits and costs, and these patients may experience significant harms when repeatedly attending the ED. Our multidisciplinary team creates individualized treatment plans for these patients. When they present to a Calgary ED/UCC, a flagged EMR document is easily accessible to treating providers. Our plans provide background and treatment recommendations for these patients, improving care and reducing repetitive, costly and potentially harmful treatments and tests. Ultimately, we improve quality of ED care regardless of site, and shift care out of the ED and into the community whenever feasible.

Implementation: A multidisciplinary team was created to select patient candidates from ED utilization data and ED/EMS staff referrals. Patients are consented to/involved with the treatment plan process whenever possible. An ED physician conducts a chart review and consults with the family physician, specialist(s) and social agencies. If there are unmet needs in the community, this is addressed with the family physician and relevant agencies. A multidisciplinary case conference occurs after which the ED physician authors a plan, which is implemented concurrent with an EMS Specialized Treatment Protocol. The team reviews and monitors all treatment plans.

We have no dedicated funding for any team members and all members participate either as volunteers or as an extension of their existing roles. Ongoing monitoring and updating of plans is increasingly labour intensive as more plans are created. Resources needed to sustain this work are protected time and funding for team members and assigned office administrative support.

Evaluation Methods: We evaluate the effectiveness of individual plans by monitoring patients' ED visit frequency. Additionally, because each plan and the needs of each patient are unique, chart reviews are frequently conducted to assess for other measures of success (i.e. Reduction in narcotics administered in ED) and unintended consequences (i.e. 72-hour readmissions). Each plan is discussed at quarterly meetings and more frequently as needed. Plans are revised as necessary, involving care providers in the community in ongoing discussions.

Regarding unintended consequences, two complaints have been raised by a single patient with a treatment plan. As a result, to ensure the patient's needs are being met, we conducted an ethics review of the plan in question and directly addressed the patient's concerns. It was determined that the concerns arose from misconceptions about the contents of her treatment plan. We have subsequently created a document for patient enrollees to ensure better understanding of the aims of the treatment plan and how to request a review, and have created a process for addressing patient concerns in the future.

Results: Preliminary data shows a significant reduction in average monthly visits across the entire group of Treatment Plan patients. Anecdotally, some patients have expressed improvements in their overall quality of life with greater support in the community and less time spent in emergency. In addition to the benefits to the system and to patients, these plans have been widely lauded by ED staff for significantly easing the care of some of our most challenging patients; this reduction in provider burn out in turn reduces the risks associated with bias when these frequent visitors are treated in the ED.

\section{Advice and Lessons Learned:}

1) Several partnerships have been key to our success: Cooperation with the patients' family physicians, consultation with the Calgary Chronic Pain Centre, and coordination with the EMS Specialized Treatment Protocol group. The ability to flag treatment plans in an EMR is a prerequisite for success, as this allows providers at each ED/UCC to easily access a plan and provide appropriate care regardless of where a patient presents. This capability has been built into our new province-wide EMR.

2) Central vetting and monitoring of plans is essential to reduce the risk of creating anchoring bias or unfairly prejudicing care providers. Plans should be written with compassionate, patient-centered language, and 
should include a statement similar to the following: "This Treatment Plan presents suggestions intended to expedite care and reduce patient harms. This plan does not preclude clinical judgement at the time of each presentation.” When disagreement or patient concerns arise, a formal ethics consultation, involving the family physician and, if relevant, chronic pain or other specialists, should be undertaken and documented on the chart.

3) Each plan should have a physician author who has conducted a deep chart review and can serve as a contact person should issues arise, but the central Treatment Plan Committee is ultimately responsible for monitoring, revising, and renewing all treatment plans. This reduces the risk of "orphaned" plans that may be out of date and could introduce harm, in case a physician author leaves the zone, retires or is otherwise unavailable. 